\title{
More expansive horizons: a review of endovascular therapy for patients with low NIHSS scores
}

\author{
David J McCarthy, ${ }^{1}$ Daniel A Tonetti, ${ }^{1}$ Jeremy Stone, ${ }^{1}$ Robert M Starke, ${ }^{2,3}$ \\ Sandra Narayanan, ${ }^{4}$ Michael J Lang, ${ }^{1}$ Ashutosh P Jadhav (1) , ${ }^{4}$ Bradley A Gross ${ }^{1}$
}

${ }^{1}$ Department of Neurosurgery, University of Pittsburgh, Pittsburgh, Pennsylvania, USA ${ }^{2}$ Department of Neurological Surgery, University of Miami MILLER School of Medicine, Miami Beach, Florida, USA ${ }^{3}$ Department of Neurosurgery, University of Miami School of Medicine, Miami, Florida, USA ${ }^{4}$ Department of Neurology, University of Pittsburgh, Pittsburgh, Pennsylvania, USA

Correspondence to Dr Bradley A Gross, Department of Neurosurgery, University of Pittsburgh, Pittsburgh, PA 15213, USA; grossb2@upmc. edu

Received 1 July 2020 Revised 8 September 2020 Accepted 13 September 2020 Published Online First 7 October 2020

\section{ABSTRACT}

While the landmark 2015 stroke trials demonstrated that endovascular therapy (EVT) was superior to medical management for the treatment of acute ischemic stroke due to large vessel occlusion, the efficacy of EVT for patients presenting with a low NIHSS score remains undetermined. We conducted a review of the EVT low National Institutes of Health Stroke Scale (NIHSS) stroke literature, identifying 24 quantitative and six qualitative publications. Details of study designs and outcome were extracted and critically discussed.

All identified qualitative studies were retrospective. There was significant study design heterogeneity, with 18 unique study designs between the 24 identified quantitative manuscripts. Study investigations included low NIHSS EVT feasibility $(n=6)$, EVT versus best medical management (BMM; $n=10)$, EVT versus intravenous therapy (IVT, n=3), and low NIHSS score versus high NIHSS score $(n=3)$. From single-arm EVT feasibility studies, the reported ranges of modified Thrombolysis in Cerebral Infarction and symptomatic intracranial hemorrhage were $78-97 \%$ and $0-10 \%$, respectively. The EVT versus BMM literature had heterogeneous results with 40\% reporting benefit with EVT and $60 \%$ reporting neutral findings. None of the studies comparing EVT with IVT reported a difference between the two revascularization therapies. The four identified meta-analyses had incongruent inclusion criteria and conflicting results. Two randomized trials are currently investigating EVT in patients with a low NIHSS score. Selected meta-analyses do suggest a potential benefit of EVT over BMM; however, current and future randomized clinical trials will better elucidate the efficacy of EVT in this patient population.

\section{INTRODUCTION}

In 2015, multiple randomized controlled clinical trials demonstrated the superiority of endovascular therapy (EVT) over medical management alone for the treatment of acute ischemic stroke (AIS) due to large vessel occlusion (LVO). ${ }^{1}$ These results were contrary to previous trials, which showed no additional benefit of EVT over IV tissue plasminogen activator alone. Importantly, these differences were attributed to many considerations, including patient selection, efficient workflow, and higher technical success. ${ }^{1}$ An exclusion criterion shared by many of the trials was mild clinical stroke severity, determined by low National Institutes of Health Stroke Scale (NIHSS) score. In trials without this exclusion criterion, patients with AIS and a low
NIHSS score were under-represented, comprising only $5 \%$ of the EVT cohorts. ${ }^{23}$ The efficacy of EVT in patients with AIS-LVO and a low NIHSS score continues to be widely debated, with the literature comprising retrospective investigations that demonstrate conflicting results. ${ }^{3-12}$

Variability in outcomes in patients with AIS and a low NIHSS score highlights the limitations of the NIHSS as a screening tool for treatment eligibility. ${ }^{13}$ While the NIHSS is predictive of overall AIS outcome, not all low NIHSS scores are equivalent with regard to clinical outcome. ${ }^{14} 15$ Sucharew et al used syndromic combination to identify two different low NIHSS profiles with distinct outcomes but both with median NIHSS score of 5 . One syndromic profile was characterized by level of consciousness and language, the other by abnormal right motor function. Patients in the latter profile demonstrated better morbidity and mortality, suggesting that the NIHSS may lack the resolution to clinically predict outcomes in low NIHSS score ranges. ${ }^{15}$ Discordant outcomes among patients with numerically low NIHSS values increase the variance of the literature-reported outcomes, making it challenging to identify the optimal treatment for these patients. While the routine use of intravenous thrombolysis (IVT) has been the standard of care since 1995, even the role of IVT for patients with a low NIHSS score remains controversial. ${ }^{16}$ Increasingly, it is recognized that the variability between patients with a low NIHSS score can be further resolved by classifying the presenting symptoms as disabling versus non-disabling. ${ }^{16} 17$

EVT in patients with a low NIHSS score and LVO is a more nuanced risk-benefit analysis than that for patients with a higher NIHSS score, with potentially smaller clinical benefit at a similar interventional risk. The greatest clinical benefit is garnered in the population subset that further declines, as deficit reversibility in this cohort may not be as feasible even in the setting of exceptional, but delayed, rescue interventional reperfusion. This must be weighed against the risk taken in patients who may recover in the absence of intervention. Investigation into the efficacy of EVT in patients with a low NIHSS score has been limited to retrospective review of prospectively collected data. Furthermore, published studies to date are limited by heterogeneous 'low NIHSS score' definitions, LVO inclusion criteria, and primary endpoints, adding to the already present outcome variability in patients with AIS and a low NIHSS score. Meta-analyses 
attempting to draw conclusions from the heterogeneous literature have consequently yielded conflicting results. ${ }^{3} 121819$ In an international survey of 607 practitioners presented with a sample patient with a low NIHSS score, the authors found significant treatment threshold disparity, with $60 \%$ deciding to pursue EVT. ${ }^{20}$ In one recent study, it was estimated that $11 \%$ of patients with LVO AIS undergoing EVT present with mild stroke symptoms. ${ }^{21}$ To aid EVT decision-making in this uncertain patient population, we present background on the natural history of patients with AIS and a low NIHSS score, and then review the 'mild stroke' EVT literature.

\section{NATURAL HISTORY OF PATIENTS WITH AIS AND A LOW NIHSS SCORE}

The Safe Implementation of Thrombolysis in Stroke (SITS) International Stroke Thrombolysis and Get with the Guidelines registries report that 21-22\% of patients with AIS present with an NIHSS score $<6$, without LVO specification. ${ }^{22} 23$ The SITS registry further reviewed vessel imaging and found that $25 \%$ of these patients with stroke and a low NIHSS score were harboring an extracranial or intracranial occlusion, resulting in an overall estimated low NIHSS AIS arterial occlusion incidence of 5\%. Documented site-specific arterial occlusion rates were $19 \%$ internal carotid artery (ICA) to ICA-terminus (ICA-T), $14.5 \% \mathrm{M} 1,23.1 \% \mathrm{M} 2,2 \%$ anterior cerebral artery (ACA), $8 \%$ posterior cerebral artery, $5 \%$ basilar, and $9.8 \%$ vertebral artery. ${ }^{23}$ With regard to EVT, the pertinent natural history discussion of low NIHSS AIS encompasses the outcomes in patients treated with conservative management $(\mathrm{CM})$ or best medical management (BMM), including IVT.

Clinical outcomes of patients with AIS and a low NIHSS score treated with CM are superior to those of patients with AIS and a high NIHSS score, but still unfavorable, with a reported $27-35 \%$ of patients dependent or dead at hospital discharge. ${ }^{13}$ 24-26 Nedeltchev et al cited a dependency rate of 23\%, though they grouped patients with a low NIHSS score with patients with rapidly improving AIS, and these two mild stroke populations may not have similar outcomes. ${ }^{27}$ When treated with IVT, the outcomes of patients with a low NIHSS score have been shown to improve if the patient has a coinciding disabling deficit, defined as preventing either basic activities of daily living or returning to work. ${ }^{1628-30}$ The PRISMS trial demonstrated that there was no added benefit of IVT in patients with a low NIHSS score presenting without a disabling deficit. ${ }^{17}$

Although a low NIHSS score is a predictor of good outcome after LVO-AIS, some of these patients may benefit from recanalization therapy. ${ }^{31}$ In conservatively managed patients with LVOAIS and a NIHSS score $\leq 8$ patients, Mokin et al retrospectively observed a $38 \%$ death or dependence rate. Outcomes did not vary significantly between occlusion location, with vessel-specific death/dependency rates of 37\% ICA, 32\% M1-M2, 59\% basilar, $45 \%$ P1-P2. ${ }^{32}$ In 378 patients with mild stroke primarily receiving $\mathrm{CM}$, Kim et al found that the presence of occlusion was independently associated with early neurologic deterioration (END; $\mathrm{OR}=2.206, \mathrm{p}=0.009) .{ }^{33}$ Similarly, Rajajee et al found that mild stroke receiving CM in patients with $\mathrm{LVO}$ is more susceptible to END (LVO 38\% vs non-LVO 3\%) and unfavorable functional outcomes (modified Rankin Scale score $(\mathrm{mRS})>2 \mathrm{LVO}$ $50 \%$ versus non-LVO $3 \%) .{ }^{34}$ In a more recent study, $19.7 \%$ of medically managed patients with LVO-AIS experienced END at a median time of 3.6 hours from hospital arrival. ${ }^{35}$ Some identified predictors of good outcome $(\mathrm{mRS}$ score $\leq 2)$ in patients with LVO-AIS and a low NIHSS score include lower NIHSS score (0-3), younger age, reperfusion therapy (IVT, EVT, or both), absence of hypertension, and absence of hyperglycemia. ${ }^{9} 3236$ Literature for the treatment of patients with LVO-AIS and a low NIHSS score with IVT has yielded conflicting results, probably related to variability in patient exclusion criteria. ${ }^{9} 173738$

\section{EVT FOR PATIENTS WITH AIS AND A LOW NIHSS SCORE Literature review methods}

A literature search was conducted on April 15, 2020 by searching the English literature in the Cochrane Library and PubMed. Using the $\mathrm{PICO}^{39}$ search method, a combination of MeSH terms and free text words were searched: ('mild stroke' OR 'mild large vessel occlusion' OR 'mild ischemic stroke' OR 'low NIHSS' OR 'low National Institutes of Health Stroke Scale') AND ('endovascular' OR 'mechanical thrombectomy' OR '(MeSH) endovascular procedures'). A total of 543 published abstracts or manuscripts were identified. Authors used the Rayyan review app to facilitate a systematic selection of articles. ${ }^{40}$ Quantitative manuscripts without a dedicated EVT arm (articles with single arm combined IVT/EVT recanalization groups) were excluded. Non-English literature and abstracts were also excluded.

Each published manuscript that met inclusion criteria and had a discrete EVT treatment group was subjected to data extraction. Owing to significant study heterogeneity and previously published meta-analyses, we did not attempt to combine patient data. Instead, we extracted the study designs and final study conclusions. Study design data points include investigative question, inclusion criteria, NIHSS definition of mild stroke, treatment details, and primary/ secondary outcomes. Single-arm EVT studies were labeled as EVT feasibility investigations. Case-control studies without IVT analyses were labeled as EVT versus BMM, whereas those with IVT specification were labeled EVT versus IVT.

\section{Literature review results}

Our literature search yielded 543 results; 30 manuscripts fulfilled our inclusion criterion, 24 quantitative $e^{3-12} 212641-52$ and six qualitative. $^{2053-58}$ Table 1 displays the identified quantitative studies with EVT treatment of patients with LVO-AIS and a low NIHSS score and their respective inclusion criteria, outcomes, and conclusions. Four meta-analyses were also identified, two of which included their original EVT patient data. There was significant study design heterogeneity, with 18 unique study designs between the 24 identified quantitative manuscripts. The largest points of disparity were study investigation, NIHSS definition of mild stroke, distal vessel exclusion criterion, and primary outcome. Study investigations included low NIHSS EVT feasibility $(n=6)$, EVT versus best medical management (BMM; $\mathrm{n}=10)$, EVT versus IVT $(\mathrm{n}=3)$, and low NIHSS score versus high NIHSS score $(n=3)$. The most common definitions of mild stroke were NIHSS score $\leq 5(n=15,63 \%)$, followed by NIHSS score $\leq 8(n=4)$, and NIHSS score $\leq 7(n=3)$. Studies with parallel investigation and mild stroke definition often had different distal vessel occlusion criteria. The most common vessel criteria used were anterior circulation ICA-terminus, middles cerebral artery (MCA)M1, or MCA-M2.

\section{EVT safety and feasibility}

Six manuscripts identified were singe-arm studies focusing on the feasibility and safety of EVT for patients with LVO-AIS and a low NIHSS score, three $(50 \%)$ of which defined low NIHSS as NIHSS score $\leq 5$. Among these single-arm trials, the technical feasibility and safety of EVT for patients with LVO-AIS and a low NIHSS score is similar to that of previous stroke trials with reported modified Thrombolysis in Cerebral Infarction (mTICI) $\geq 2 b$ and symptomatic intracranial hemorrhage (sICH) rates ranging from $78 \%$ to $97 \%$ and 
Table 1 Identified low NIHSS EVT literature

\begin{tabular}{|c|c|c|c|c|c|c|c|}
\hline Date & Author & Investigation & $\mathrm{N}$ & NIHSS score & *Vessel criteria* & Primary outcome & Study conclusion \\
\hline 2020 & Toth et $a l^{41}$ & EVT feasibility & 20 & $\leq 5$ & $\begin{array}{l}\text { Anterior and posterior } \mathrm{M} 2 \text {, } \\
\text { basilar }\end{array}$ & $\mathrm{mRS} \leq 1$ & $\begin{array}{l}95 \% \mathrm{mTICl} \geq 2 \mathrm{~b} \\
95 \% \mathrm{mRS} \leq 1 \\
0 \% \text { sICH, } 5 \% \text { SND }\end{array}$ \\
\hline 2016 & Bhogal et $a l^{42}$ & EVT feasibility & 41 & $\leq 5$ & M1 only & $\mathrm{mTICl} \geq 2 \mathrm{~b}$ & $\begin{array}{l}75 \% \mathrm{mRS} \leq 2 \\
88 \% \mathrm{mTICl} \geq 2 \mathrm{~b} \\
10 \% \mathrm{sICH}\end{array}$ \\
\hline 2018 & Kaschner et al ${ }^{43}$ & EVT feasibility & 30 & $\leq 5$ & M1 only & $m R S \leq 2$ & $\begin{array}{l}97 \% \mathrm{mTICl} \geq 2 \mathrm{~b} \\
3 \% \mathrm{sICH}\end{array}$ \\
\hline 2017 & Dargazanli et $a^{44}$ & EVT feasibility & 138 & $\leq 7$ & Anterior M2, ICA-T & $\mathrm{mTICl} \geq 2 \mathrm{~b}$ & $\begin{array}{l}\mathrm{mTICl} \geq 2 \mathrm{~b} \text { predictive of } \mathrm{FI} \\
81 \% \mathrm{mTICl} \geq 2 \mathrm{~b}\end{array}$ \\
\hline 2016 & Pfaff et al ${ }^{45}$ & EVT feasibility & 33 & $\leq 8$ & None & $\mathrm{mTICl} \geq 2 \mathrm{~b}$ & $\begin{array}{l}\text { EVT is safe } \\
78 \% \mathrm{mTICl} \geq 2 \mathrm{~b} \\
6 \% \mathrm{sICH}\end{array}$ \\
\hline 2018 & Bowen et $a l^{46}$ & EVT feasibility & 72 & $\leq 8$ & None & $\mathrm{mRS} \leq 2$ & $\begin{array}{l}\text { EVT is safe } \\
93 \% \mathrm{mTICl} \geq 2 \mathrm{~b} \\
0 \% \mathrm{sICH}\end{array}$ \\
\hline 2014 & Urra et al ${ }^{4}$ & EVT vs BMM & 78 & $\leq 5$ & $\begin{array}{l}\text { Anterior and posterior } \mathrm{M} 2 \text {, } \\
\mathrm{A} 1 \text {, basilar, } \mathrm{P} 1\end{array}$ & $\mathrm{mRS} \leq 1$ & NS \\
\hline 2018 & Haussen et al ${ }^{5}$ & EVT vs BMM & 118 & $\leq 5$ & $\begin{array}{l}\text { Anterior and posterior } \mathrm{M} 2 \text {, } \\
\text { ACA, basilar }\end{array}$ & NIHSS shift & EVT benefit $(p=0.03)$ \\
\hline 2018 & Nagel et al ${ }^{6}$ & EVT vs BMM & 300 & $\leq 5$ & $\begin{array}{l}\text { Anterior and posterior } \mathrm{M} 2 \text {, } \\
\mathrm{ACA} \text {, basilar }\end{array}$ & $\mathrm{mRS} \leq 2$ & EVT benefit $(p=0.03)$ \\
\hline 2019 & tGoyal et al $l^{3}$ & EVT vs BMM & 251 & $\leq 5$ & Anterior M2, ICA-T & $\mathrm{mRS} \leq 2$ & NS \\
\hline 2017 & Haussen et al ${ }^{7}$ & EVT vs BMM & 32 & $\leq 5$ & Anterior M2, ICA-T & NIHSS shift & EVT benefit $(p=0.04)$ \\
\hline 2018 & Sarraj et $a l^{8}$ & EVT vs BMM & 214 & $\leq 5$ & Anterior M4, ACA & $\mathrm{mRS} \leq 1$ & NS \\
\hline 2020 & Saito et $a l^{9}$ & EVT vs BMM & 272 & $\leq 5$ & $\begin{array}{l}\text { Anterior and posterior } \mathrm{M} 3 \text {, } \\
\mathrm{A} 2, \mathrm{P} 2\end{array}$ & $\mathrm{mRS} \leq 2$ & NS \\
\hline 2020 & Wolman et al ${ }^{10}$ & EVT vs BMM & 47 & $\leq 6$ & Anterior-none & NIHSS shift & NS \\
\hline 2017 & Dargazanli et al ${ }^{11}$ & EVT vs BMM & 301 & $\leq 7$ & Anterior M2, ICA-T & $m R S \leq 1$ & NS \\
\hline 2019 & †Shang et al ${ }^{12}$ & EVT vs BMM & 177 & $\leq 8$ & Anterior $\mathrm{M} 2, \mathrm{ACA}$ & $\mathrm{mRS} \leq 1$ & EVT benefit $(p=0.008)$ \\
\hline 2019 & Manno et $a l^{47}$ & EVT vs IVT & 312 & $\leq 5$ & Anterior M2, ICA-T & $\mathrm{mRS} \leq 1$ & NS \\
\hline 2017 & Messer et $a l^{48}$ & EVT vs IVT & 54 & $\leq 5$ & Anterior M2, ICA-T & $\mathrm{mRS} \leq 1$ & NS \\
\hline 2018 & Kastrup et al ${ }^{49}$ & EVT vs IVT & 305 & $\leq 10$ & Anterior M2, ICA-T & $\mathrm{mRS} \leq 2$ & NS \\
\hline 2020 & Heldner et $\left.a\right|^{50}$ & EVT vs IVT vs CM & 185 & $\leq 5$ & Anterior M1, ICA-T & SND & $\begin{array}{l}\text { EVT or IVT better than } \\
\text { conservative }\end{array}$ \\
\hline 2018 & Shang et $a l^{51}$ & Extended window & 93 & $\leq 8$ & Anterior $\mathrm{M} 2, \mathrm{ACA}$ & $\mathrm{mRS} \leq 1$ & NS \\
\hline 2019 & Goldhoorn et $a l^{26}$ & Low vs high NIHSS score & 71 & $\leq 5$ & Anterior $\mathrm{M} 2, \mathrm{~A} 2$ & $\mathrm{mRS} \leq 2$ & $\begin{array}{l}\text { Outcomes better patients } \\
\text { within low NIHSS score }\end{array}$ \\
\hline 2019 & Kaesmacher et al ${ }^{52}$ & Low vs high NIHSS score & 193 & $\leq 7$ & $\begin{array}{l}\text { Anterior and posterior } \mathrm{M} 3 \text {, } \\
\mathrm{A} 2, \mathrm{P} 2\end{array}$ & $\mathrm{mRS} \leq 1$ & Safety/efficacy similar \\
\hline 2019 & Asdaghi et $a l^{21}$ & Low vs high NIHSS score & 446 & $\leq 5$ & None & Patient characteristics & - \\
\hline
\end{tabular}

*For vessel imaging criteria the most distal artery of the parent artery is listed. For example, if M4 is listed the study included MCA sections M1, M2, M3, and M4.

tIndicates study also conducted meta-analysis of literature.

ACA, anterior cerebral artery; BMM, best medical management; CM, conservative management; EVT, endovascular therapy; ICA-T, internal carotid artery-terminus; IVT, intravenous therapy; MCA, middle cerebral artery; mRS, mofified Rankin Scale; $\mathrm{mTICl}$, modified Thrombolysis in Cerebral Infarction; N, number of patients; NS, no difference in primary outcome; sICH, symptomatic intracranial hemorrhage; SND, symptomatic neurological deficit.

$0 \%$ to $10 \%$, respectively. ${ }^{24-46}$ Many studies found that successful recanalization was significantly predictive of favorable clinical outcome. ${ }^{9444652}$ Patients with NIHSS score $\leq 7$ reported excellent outcome (mRS score $\leq 1)$; rates for mTICI scores $<2 b, 2 b$, and 3 were $35 \%, 62 \%$, and $79 \%$, respectively. ${ }^{44}$ With a NIHSS score $\leq 5$ upper limit, functional independence (mRS score $\leq 2$ ) rates were $89 \%$ in patients with $\mathrm{mTICI} \geq 2 \mathrm{~b}$ versus $60 \%$ in those not recanalized $(\mathrm{p}<0.05)^{9}{ }^{9}$

\section{EVT vs best medical management}

We identified 10 manuscripts comparing EVT with BMM in patients with LVO-AIS and a low NIHSS score (table 2), six (60\%) of which had low NIHSS defined as NIHSS score $\leq 5{ }^{3-12}$ Additionally, four meta-analyses were identified (two, NIHSS score $\leq 5$; two, NIHSS score $\leq 8) .{ }^{3121819}$ To compare EVT with BMM in mild strokes, the functional benefits of recanalization must be weighed against the added risk of the EVT procedure.

Four of the EVT versus BMM manuscripts concluded that EVT provided clinical benefit. ${ }^{5-712}$ Outcome endpoints in these investigations were the shift in NIHSS score (discharge NIHSS score minus admission NIHSS score) and mRS score at 90-day follow-up. In 32 patients with LVO-AIS and NIHSS score $\leq 5$, Haussen et al observed that immediate EVT resulted in a better discharge NIHSS shift than for those treated with BMM $(-2.5$ vs $0 ; \mathrm{p}<0.01)$. However, 90-day functional independence and mortality rates between the two cohorts were the same. ${ }^{7}$ Subsequently, Haussen et al externally validated and expanded their 
Table 2 Results of studies investigating endovascular therapy versus best medical management

\begin{tabular}{|c|c|c|c|c|c|c|c|c|c|c|c|c|}
\hline \multirow[b]{2}{*}{ Year } & \multirow[b]{2}{*}{ Author } & \multirow{2}{*}{$\begin{array}{l}\text { IVT in } \\
\text { EVT arm }\end{array}$} & \multicolumn{2}{|c|}{ EVT $m R S \leq 1$} & \multicolumn{2}{|c|}{ EVT $m R S \leq 2$} & \multicolumn{2}{|c|}{ NIHSS shift with EVT } & \multicolumn{2}{|c|}{ sICH } & \multicolumn{2}{|c|}{$\mathrm{alCH}$} \\
\hline & & & aOR & $\mathbf{P}$ & $\mathrm{aOR}$ & $\mathbf{P}$ & Shift & $\mathbf{P}$ & EVT $\%$ & BMM \% & EVT $\%$ & ВМM \% \\
\hline 2014 & Urra et $a l^{4}$ & $47 \%$ & NS & NS & NS & NS & - & - & $11.8^{*}$ & 0 * & - & - \\
\hline 2018 & Haussen et $a l^{5}$ & $31 \%$ & - & - & $-21 \%$ & $<0.01$ & -3.74 & 0.016 & 6.7 & 0 & - & - \\
\hline 2018 & Nagel et a ${ }^{6}$ & $51 \%$ & NS & NS & 3.1 & $\mathrm{NR}^{*}$ & - & - & 5.2 & 2.6 & - & - \\
\hline 2020 & ${ }^{*}$ Goyal et $a l^{3}$ & $54 \%$ & 0.72 & 0.47 & 0.73 & 0.64 & - & - & 4.4 & 0.9 & $22^{*}$ & $3^{*}$ \\
\hline 2017 & Haussen et $a l^{7}$ & $60 \%$ & - & - & - & - & -2.5 & 0.01 & 0 & 0 & - & - \\
\hline 2018 & Sarraj et a ${ }^{8}$ & $31 \%$ & 1.3 & 0.47 & 0.9 & 0.77 & - & - & $5.8^{*}$ & $0^{*}$ & 5.3 & 10 \\
\hline 2020 & Saito et $a l^{9}$ & $9.1 \%$ & NS & NS & 1.65 & 0.25 & - & - & NS & NS & NS & NS \\
\hline 2020 & Wolman et al ${ }^{10}$ & $35 \%$ & - & - & - & - & -0.8 & 0.62 & NS & NS & $35^{*}$ & $10^{*}$ \\
\hline 2017 & Dargazanli et al ${ }^{11}$ & $61 \%$ & 1.15 & NS & NS & NS & - & - & NS & NS & $16.5^{*}$ & $6.1^{*}$ \\
\hline 2019 & *Shang et al ${ }^{12}$ & $29 \%$ & NS & NS & 3.2 & 0.008 & - & - & $10^{*}$ & $2^{*}$ & - & - \\
\hline
\end{tabular}

- Analysis not performed.

*Statistically significant $(\mathrm{p}<0.05)$ but numbers either not reported or bivariate comparisons.

alCH, any intracranial hemorrhage or in some cases asymptomatic; aOR, adjusted OR without imputation; EVT, endovascular therapy; IVT, intravascular thrombolysis; mRS, modified Rankin Scale; NIHSS, National Institutes of Health Stroke Scale; NR, not reported value; NS, not significant with specific number not reported; sICH, symptomatic intracranial hemorrhage.

findings in a multicenter cohort study, in which EVT was an independent predictor of lower discharge NIHSS score $(p=0.04)$, favorable NIHSS shift $(p=0.03)$, and increased independence rates at discharge $(p=0.03){ }^{5}$ While NIHSS shift has been used in non-EVT low NIHSS AIS literature, few EVT low NIHSS investigations used NIHSS shift as a primary endpoint, making it difficult to cross-validate their findings with other studies. 571034 Only one other EVT versus BMM study with an NIHSS score $\leq 5$ criterion detected an EVT benefit. To minimize treatment selection bias, Nagel et al matched patients with a low NIHSS score, receiving EVT and BMM, based on age, baseline NIHSS score, and occlusion site. Patients receiving EVT had higher rates of favorable outcome (mRS score $\leq 2$, OR $=3.1 ; 84.4 \%$ vs $70.1 \% ; \mathrm{p}=0.03) .^{6}$ In patients with an NIHSS score $\leq 8$, Shang et al found that EVT resulted in a significantly higher likelihood of excellent outcome $(\mathrm{OR}=3.2, \mathrm{p}=0.008)$; however, the metaanalysis they performed failed to confirm these associations. ${ }^{12}$

Six manuscripts with original patient data failed to detect EVT clinical benefit in patients with a low NIHSS score. ${ }^{34} 8-11$ Urra et al published the first retrospective study of low NIHSS EVT versus BMM in 2014. In 78 patients they observed no EVT benefit; however, IV thrombolysis was predictive of full recovery $(\mathrm{OR}=3.7 ; \mathrm{p}=0.015)$. There was numerically more IVT administration in the BMM group that EVT group, though this was not significant $(66 \%$ vs $47 \%$; $=0.09) .{ }^{4}$ Sarraj et al also observed no overall benefit for EVT. However, when stratifying by vessel location, patients with MCA-M1 LVO location trended towards higher rates of excellent outcome $(\mathrm{OR}=3.3, \mathrm{p}=0.07){ }^{8}$ Additional EVT versus BMM articles failed to detect clinical benefit for EVT. ${ }^{9-11}$ Recently, Goyal et al performed a meta-analysis of patients with AIS and NIHSS score $\leq 5$ with an additional 251 patients from 16 centers. ${ }^{3}$ From their original data, patients receiving BMM and EVT had similar rates of 90-day functional outcomes (mRS score $\leq 2,76.7 \%$ vs $85.2 \%, p=0.12$; mRS score $\leq 1,63.1 \%$ vs $70.4 \%, p=0.26$ ). After adjusting for confounders and using imputation for missing follow-up data, EVT had a lower likelihood of a 90-day mRS score $\leq 2(\mathrm{OR}=0.42, \mathrm{p}=0.04)$. Interestingly, this association disappeared when missing data were not extrapolated $(\mathrm{OR}=0.72, \mathrm{p}=0.47)$, highlighting the need for higher-quality data in this patient population.

Hemorrhagic outcomes were closely linked with clinical outcomes, with three of four studies that showed benefit from EVT observing no hemorrhagic differences and five of six studies without benefit from EVT reporting increased hemorrhage in patients receiving EVT. Three investigations (30\%) observed significantly higher rates of $\mathrm{sICH}$ in patients receiving EVT $(11.8$ vs $0 \%, \mathrm{p}=0.03),{ }^{4}(5.8 \%$ vs $0 \% ; \mathrm{p}=0.02),{ }^{8}(10 \%$ vs $2 \%$; $\mathrm{p}=0.02) .{ }^{12}$ However, the increased sICH risk observed by Shang et al was not significant after adjusting for confounders. ${ }^{12}$ Three studies (30\%) observed increased EVT asymptomatic intracranial hemorrhage $(35 \%$ vs $10 \%, p=0.04),{ }^{10}(22 \%$ vs $3 \%, p=0.002),{ }^{3}$ $(16.5 \% \text { vs } 6.1 \%, \mathrm{p}=0.008)^{11}$ but not sICH. ${ }^{310} 11$ Four investigations (40\%) did not detect any difference in hemorrhagic complications between EVT and BMM..$^{5-79}$

We identified four meta-analyses for EVT versus BMM, two of which concluded EVT benefit. ${ }^{3} 121819$ Unfortunately, these analyses are challenging to compare owing to heterogeneous inclusion criteria and incongruous time of literature review.

\section{EVT vs IVT}

In patients with LVO-AIS and normal NIHSS ranges, EVT with or without IVT is superior to IVT alone, and EVT alone is noninferior to EVT + IVT, although, the same may not hold true in patients with a low NIHSS score. ${ }^{259}$ The low NIHSS LVOAIS literature, with an admixture of revascularization cohorts (EVT or IVT), reports that any reperfusion therapy (IVT, EVT, or both) in patients with LVO-AIS is associated with increased functional independence $(O R=3.1, p=0.030){ }^{36}$ This suggests that both IVT and EVT play a role in improving outcomes. Our review of the EVT versus BMM literature yielded EVT bridging rates ranging from $9 \%$ to $61 \%$, with only one study performing IVT subgroup analyses (table 2). ${ }^{9}$ This wide IVT treatment range probably increases interstudy outcome variability. It is essential to compare IVT with EVT \pm IVT in patients with a low NIHSS score to elucidate the role of EVT bridging therapy in IVTeligible patients. Our literature review identified four studies that compared EVT with IVT. ${ }^{47-50}$

Two studies had dedicated EVT alone analyses. ${ }^{47} 50$ In a propensity matched analysis, Manno et al compared patients with LVO-AIS and NIHSS score $\leq 5$ treated with EVT \pm IVT with IVT alone; both resulted in comparable mRS $\leq 1$ rates $(63 \%$ vs $65.7 \%$, respectively; $p=0.840)$. In vessel-specific subgroup analyses, there remained no difference (ICA/M1 p=0.9, M2 p=0.8). Interestingly, they observed a trend towards higher mortality in patients receiving EVT $(9.3 \%$ vs $2.8 \% ; \mathrm{p}=0.06)$ even though sICH was rare in both groups $(2.8 \%$ vs $0 \% ; \mathrm{p}=0.997)$. When 


\begin{tabular}{|c|c|}
\hline Total number of studies & 24 \\
\hline \multicolumn{2}{|l|}{ Low NIHSS definitions } \\
\hline$\leq 5$ & $15(63 \%)$ \\
\hline$\leq 7$ & $3(13 \%)$ \\
\hline$\leq 8$ & $4(17 \%)$ \\
\hline Other & $2(8 \%)$ \\
\hline \multicolumn{2}{|l|}{ EVT feasibility studies (from six studies) } \\
\hline $\mathrm{mTICl} \leq 2 \mathrm{~b}$ & $78-97 \%$ \\
\hline $\mathrm{sICH}$ & $0-10 \%$ \\
\hline \multicolumn{2}{|l|}{ EVT vs BMM } \\
\hline Primary outcome showed EVT benefit & 4 of 10 studies \\
\hline sICH EVT risk significant & 6 of 10 studies \\
\hline \multicolumn{2}{|l|}{ EVT vs IVT } \\
\hline Primary outcome showed EVT benefit & 0 of 4 studies \\
\hline sICH EVT risk significant & 0 of 4 studies \\
\hline
\end{tabular}

BMM, best medical management; EVT, endovascular therapy; IVT, intravascular thrombolysis; mTICl, modified Thrombolysis in Cerebral Infarction; NIHSS, National Institutes of Health Stroke Scale; sICH, symptomatic intracranial hemorrhage.

treatment groups were separated into EVT alone, EVT with IVT, and IVT alone, they observed an association between EVT only and mortality (OR $12.75, \mathrm{p}=0.004)$, suggesting that EVT alone may incur an additional non-hemorrhagic risk. Retrospective comparisons between these groups are flawed owing to the longer onset to recanalization times in IVT-ineligible patients. ${ }^{47}$ Heldner et al also observed no difference between patients with LVO and a low NIHSS score treated with EVT alone and IVT alone. ${ }^{50}$ Similarly, the two other studies comparing EVT \pm IVT with IVT alone demonstrated no significant difference in outcomes between treatments. ${ }^{48} 49$ It is possible that EVT may be efficacious only in IVT-ineligible patients or that only one thrombolytic treatment is necessary in this patient population.

\section{EVT patient selection}

The importance of patient selection in patients with LVO-AIS has been repeatedly demonstrated in past EVT trials. ${ }^{12}$ Based on the conflicting literature results which are summarized in table 3 , we hypothesize that EVT is likely beneficial for select patients with a low NIHSS score, although, the challenge will lie in identifying which patients will benefit. Factors of particular consideration include elevated blood pressure on admission, a positive heads-up test, and a large radiographic perfusion deficit, as ideal low NIHSS EVT candidates are those who will develop END. A reported range of $18 \%$ to $34.6 \%$ of patients with LVO and a low NIHSS score progress to END. ${ }^{1160}$ Unfortunately, delaying EVT until clinical manifestation of END is apparent does not result in favorable outcomes and may incur more risk. ${ }^{6} 7104850$ Nagel et al observed that patients who received 'rescue' EVT had worse outcomes than those who received BMM or immediate EVT (54.5\% rescue EVT, 71.7\% BMM, alone, 85\% immediate $\mathrm{EVTp}=0.007) .^{6}$

Patients with LVO-AIS and a low NIHSS score with MCA-M1 or ICA occlusions are more likely to develop END $(p=0.04)$, supporting the applicability of distal vessel exclusion criteria. ${ }^{60}$ Patients with proximal LVO theoretically have a larger area of brain at risk, and may have a wider EVT benefit margin. ${ }^{8}$ Furthermore, distal LVO EVT may incur a higher procedural risk of vessel perforation. A large source of our observed study heterogeneity was due to distal vessel exclusion criteria. A few EVT feasibility studies claimed that their high revascularization rates were a result of including only MCA-M1 LVO. ${ }^{44} 52$ However, these studies were limited by a small sample size. While Sarraj et al observed no overall benefit for EVT, a sensitivity analysis demonstrated that EVT trended towards significance for MCA-M1 LVO (mRS score $\leq 1, \mathrm{OR}=3.3, \mathrm{p}=0.07$ ). Distal LVO did not reflect this trend: M2 (mRS score $\leq 1,64.3 \%$ EVT vs 72.7\% BMM) and M3/M4/ACA (0\% EVT vs 53.8\% BMM). ${ }^{8}$

Additional patient selection considerations include timing and patient collateral status. Patients who present late from onset of symptoms ( $>6$ hours) have an already narrowed time window in which to develop END, whereas those who present early have a more uncertain clinical course. For patients who are beyond the IVT treatment window, Shang et al demonstrated that EVT in patients with a low NIHSS score is feasible up to 24 hours following symptom onset; however, there was no functional improvement observed with EVT. ${ }^{51}$ Robust collateral status has been correlated with reduced infarct expansion and favorable outcomes in LVO-AIS. ${ }^{61}$ It is likely that low patients with LVO and a low NIHSS score with poor collateral status are more susceptible to END. This requires further investigation, but it highlights the utility of perfusion mapping in this patient population. Ultimately, randomized clinical trials will be essential to elucidate the proper patient selection for EVT in patients with LVO-AIS and a low NIHSS score.

\section{Ongoing trials}

Importantly, the majority of medically managed patients with LVO and a low NIHSS score will not progress to END, making it challenging for future studies to detect EVT efficacy in the entire population. In order to adequately assess EVT efficacy, studies will require either a large sample size or stringent inclusion criteria. Our literature review yielded two ongoing clinical trials investigating the efficacy of EVT in patients with LVO-AIS and a low NIHSS score: "Endovascular Therapy for Low NIHSS Ischemic Strokes" (ENDOLOW) and "Minor Stroke Therapy Evaluation" (MOSTE). ${ }^{534}$ Both trials are enrolling patients with NIHSS score $\leq 5$ with proximal anterior LVO (ICA, MCA-M1). ENDOLOW is a North American based trial that planned to enroll 200 patients presenting within 8 hours of symptom onset, randomizing to either EVT with EmboTrap II or BMM. MOSTE is a European based trial that planned to recruit 824 patients presenting within 24 hours of symptom onset, randomizing to EVT or BMM. Major endpoints of the trials include mRS shift, mRS score $\leq 1$ rate, $\mathrm{mRS}$ score $\leq 2$ rate, sICH, END (increase in NIHSS score of $\geq 4$ ), and health-related quality of life. The broad inclusion criteria and large enrollment size in the MOSTE trial is probably aimed at specifying which patients will benefit from EVT. The ENDOLOW trial is aimed at quickly determining EVT efficacy in smaller subset of patients.

\section{Limitations}

Our review is comprehensive, but it precludes data synthesis and aggregate calculations. Additionally, our summary of the natural history of patients with a low NIHSS score was not performed systematically.

\section{CONCLUSION}

Our understanding of the role of endovascular therapy in patients with acute ischemic stroke with LVO who present with a low NIHSS score is evolving. Current literature is limited to retrospective reviews that have heterogeneous study 
designs and inclusion criteria and report conflicting results. Selected meta-analyses do suggest a potential benefit of EVT over BMM. Selection of patients with a low NIHSS score for EVT will be imperative and may incorporate occlusion location, symptom onset to presentation time, and patient collateral status. Unlike the current literature, ongoing trials have contiguous definitions of low NIHSS scores and outcome measures. Current and future randomized clinical trials will better elucidate the safety and efficacy of endovascular therapy in this patient population.

Twitter Robert M Starke @Starke_neurosurgery, Sandra Narayanan @ SandraNarayanan and Ashutosh P Jadhav @ashupjadhav

Contributors Drafting the article: DJM, BAG. Acquisition of data/data analysis: DJM, APJ, BAG. Reviewed and revised article prior to submission: All authors. Study supervision: BAG.

Funding The authors have not declared a specific grant for this research from any funding agency in the public, commercial or not-for-profit sectors.

Competing interests BAG: Consultant: Microvention, Medtronic. RMS

Consultant: Penumbra, Abbott, Medtronic, InNeuroCo, Cerenovus.

Patient consent for publication Not required.

Provenance and peer review Commissioned; externally peer reviewed.

ORCID iD

Ashutosh P Jadhav http://orcid.org/0000-0002-9454-0678

\section{REFERENCES}

Broderick JP, Palesch YY, Demchuk AM, et al. Endovascular therapy after intravenous t-PA versus t-PA alone for stroke. N Engl J Med 2013;368:893-903.

2 Goyal M, Menon BK, van Zwam WH, et al. Endovascular thrombectomy after large-vessel ischaemic stroke: a meta-analysis of individual patient data from five randomised trials. Lancet 2016;387:1723-31.

3 Goyal N, Tsivgoulis G, Malhotra K, et al. Medical management vs mechanical thrombectomy for mild strokes: an international multicenter study and systematic review and meta-analysis. JAMA Neurol 2020;77:16-24.

4 Urra X, San Román L, Gil F, et al. Medical and endovascular treatment of patients with large vessel occlusion presenting with mild symptoms: an observational multicenter study. Cerebrovasc Dis 2014;38:418-24.

5 Haussen DC, Lima FO, Bouslama M, et al. Thrombectomy versus medical management for large vessel occlusion strokes with minimal symptoms: an analysis from STOPStroke and GESTOR cohorts. J Neurointerv Surg 2018:10:325-9.

6 Nagel S, Bouslama M, Krause LU, et al. Mechanical thrombectomy in patients with milder strokes and large vessel occlusions. Stroke 2018;49:2391-7.

7 Haussen DC, Bouslama M, Grossberg JA, et al. Too good to intervene? Thrombectomy for large vessel occlusion strokes with minimal symptoms: an intention-to-treat analysis. J Neurointerv Surg 2017:9:917-21.

8 Sarraj A, Hassan A, Savitz SI, et al. Endovascular thrombectomy for mild strokes: how low should we go? Stroke 2018;49:2398-405.

9 Saito T, Itabashi R, Yazawa Y, et al. Clinical outcome of patients with large vessel occlusion and low National Institutes of Health Stroke Scale scores: subanalysis of the RESCUE-Japan registry 2. Stroke 2020;51:1458-63.

10 Wolman DN, Marcellus DG, Lansberg MG, et al. Endovascular versus medical therapy for large-vessel anterior occlusive stroke presenting with mild symptoms. Int I Stroke 2020;15:324-31.

11 Dargazanli C, Arquizan C, Gory B, et al. Mechanical thrombectomy for minor and mild stroke patients harboring large vessel occlusion in the anterior circulation: a multicenter cohort study. Stroke 2017;48:3274-81.

12 Shang X-J, Shi Z-H, He C-F, et al. Efficacy and safety of endovascular thrombectomy in mild ischemic stroke: results from a retrospective study and meta-analysis of previous trials. BMC Neurol 2019;19:150

13 Kenmuir CL, Hammer M, Jovin T, et al. Predictors of outcome in patients presenting with acute ischemic stroke and mild stroke scale scores. J Stroke Cerebrovasc Dis 2015;24:1685-9.

14 Adams HP, Davis PH, Leira EC, et al. Baseline NIH stroke scale score strongly predicts outcome after stroke: a report of the trial of ORG 10172 in acute stroke treatment (TOAST). Neurology 1999;53:126-31.

15 Sucharew H, Khoury J, Moomaw CJ, et al. Profiles of the National Institutes of Health Stroke Scale items as a predictor of patient outcome. Stroke 2013:44:2182-7.

16 Powers WJ, Rabinstein AA, Ackerson T, et al. 2018 guidelines for the early management of patients with acute ischemic stroke: a guideline for healthcare professionals from the American Heart Association/American Stroke Association. Stroke 2018;49:e46-99.
17 Khatri P, Kleindorfer DO, Devlin T, et al. Effect of alteplase vs aspirin on functional outcome for patients with acute ischemic stroke and minor nondisabling neurologic deficits: the PRISMS randomized clinical trial. JAMA 2018;320:156-66.

18 Griessenauer CJ, Medin C, Maingard J, et al. Endovascular mechanical thrombectomy in large-vessel occlusion ischemic stroke presenting with low National Institutes of Health Stroke Scale: systematic review and meta-analysis. World Neurosurg 2018;110:263-9

19 Xiong Y-J, Gong J-M, Zhang Y-C, et al. Endovascular thrombectomy versus medical treatment for large vessel occlusion stroke with mild symptoms: a meta-analysis. PLoS One 2018;13:e0203066.

20 Ospel JM, Kim B, Heo J-H, et al. Endovascular treatment decision-making in acute ischemic stroke patients with large vessel occlusion and low National Institutes of Health Stroke Scale: insights from UNMASK EVT, an international multidisciplinary survey. Neuroradiology 2020;62:715-21.

21 Asdaghi N, Yavagal DR, Wang K, et al. Patterns and outcomes of endovascular therapy in mild stroke. Stroke 2019;50:2101-7.

22 Romano JG, Smith EE, Liang L, et al. Outcomes in mild acute ischemic stroke treated with intravenous thrombolysis: a retrospective analysis of the Get With the Guidelines-Stroke Registry. JAMA Neurol 2015;72:423-31.

23 Mazya MV, Cooray C, Lees KR, et al. Minor stroke due to large artery occlusion. when is intravenous thrombolysis not enough? results from the SITS international stroke thrombolysis register. Eur Stroke J 2018;3:29-38.

24 Barber PA, Zhang J, Demchuk AM, et al. Why are stroke patients excluded from TPA therapy? An analysis of patient eligibility. Neurology 2001;56:1015-20.

25 Smith EE, Abdullah AR, Petkovska I, et al. Poor outcomes in patients who do not receive intravenous tissue plasminogen activator because of mild or improving ischemic stroke. Stroke 2005;36:2497-9.

26 Goldhoorn RB, Mulder MJHL, Jansen IGH, et al. Safety and outcome of endovascular treatment for minor ischemic stroke: results from the multicenter clinical registry of endovascular treatment of acute ischemic stroke in the Netherlands. I Stroke Cerebrovasc Dis 2019;28:542-9.

27 Nedeltchev K, Schwegler B, Haefeli T, et al. Outcome of stroke with mild or rapidly improving symptoms. Stroke 2007;38:2531-5.

28 National Institute of Neurological Disorders Stroke rt-PA Stroke Study Group. Recombinant tissue plasminogen activator for minor strokes: the National Institute of Neurological Disorders and Stroke rt-PA stroke study experience. Ann Emerg Med 2005;46:243-52.

29 Urra X, Ariño H, Llull L, et al. The outcome of patients with mild stroke improves after treatment with systemic thrombolysis. PLoS One 2013:8:e59420

30 Köhrmann M, Nowe T, Huttner HB, et al. Safety and outcome after thrombolysis in stroke patients with mild symptoms. Cerebrovasc Dis 2009;27:160-6.

31 Lima FO, Furie KL, Silva GS, et al. Prognosis of untreated strokes due to anterior circulation proximal intracranial arterial occlusions detected by use of computed tomography angiography. JAMA Neurol 2014;71:151-7.

32 Mokin M, Masud MW, Dumont TM, et al. Outcomes in patients with acute ischemic stroke from proximal intracranial vessel occlusion and NIHSS score below 8. J Neurointerv Surg 2014;6:413-7.

33 Kim J-T, Park M-S, Chang J, et al. Proximal arterial occlusion in acute ischemic stroke with low NIHSS scores should not be considered as mild stroke. PLoS One 2013:8:e70996

34 Rajajee V, Kidwell C, Starkman S, et al. Early MRI and outcomes of untreated patients with mild or improving ischemic stroke. Neurology 2006;67:980-4.

35 Saleem Y, Nogueira RG, Rodrigues GM, et al. Acute neurological deterioration in large vessel occlusions and mild symptoms managed medically. Stroke 2020;51:1428-34.

36 Heldner MR, Jung S, Zubler C, et al. Outcome of patients with occlusions of the internal carotid artery or the main stem of the middle cerebral artery with NIHSS score of less than 5: comparison between thrombolysed and non-thrombolysed patients. J Neurol Neurosurg Psychiatry 2015;86:755-60.

37 Urra X, Ariño H, Llull L, et al. The outcome of patients with mild stroke improves after treatment with systemic thrombolysis. PLoS One 2013;8:e59420.

38 Zhu W, Churilov L, Campbell BCV, et al. Does large vessel occlusion affect clinical outcome in stroke with mild neurologic deficits after intravenous thrombolysis? J Stroke Cerebrovasc Dis 2014:23:2888-93.

39 Schardt C, Adams MB, Owens T, et al. Utilization of the PICO framework to improve searching PubMed for clinical questions. BMC Med Inform Decis Mak 2007;7:16

40 Ouzzani M, Hammady H, Fedorowicz Z, et al. Rayyan-a web and mobile APP for systematic reviews. Syst Rev 2016;5:210.

41 Toth G, Ortega-Gutierrez S, Tsai JP, et al. The safety and feasibility of mechanical thrombectomy for mild acute ischemic stroke with large vessel occlusion. Neurosurgery 2020:86:802-7.

42 Bhogal P, Bücke P, Ganslandt 0, et al. Mechanical thrombectomy in patients with M1 occlusion and NIHSS score $\leq 5$ : a single-centre experience. Stroke Vasc Neurol 2016;1:165-71.

43 Kaschner MG, Caspers J, Rubbert C, et al. Mechanical thrombectomy in MCAmainstem occlusion in patients with low NIHSS scores. Interv Neuroradiol 2018;24:398-404.

44 Dargazanli C, Consoli A, Gory B, et al. Is reperfusion useful in ischaemic stroke patients presenting with a low National Institutes of Health Stroke Scale and 
a proximal large vessel occlusion of the anterior circulation? Cerebrovasc Dis 2017:43:305-12.

45 Pfaff J, Herweh C, Pham M, et al. Mechanical thrombectomy in patients with acute ischemic stroke and lower NIHSS scores: recanalization rates, periprocedural complications, and clinical outcome. AJNR Am J Neuroradiol 2016;37:2066-71.

46 Bowen MT, Rebello LC, Bouslama M, et al. Clinical and imaging outcomes of endovascular therapy in patients with acute large vessel occlusion stroke and mild clinical symptoms. Interv Neurol 2018;7:91-8.

47 Manno C, Disanto G, Bianco G, et al. Outcome of endovascular therapy in stroke with large vessel occlusion and mild symptoms. Neurology 2019;93:10.1212/ WNL.0000000000008362-26.

48 Messer MP, Schönenberger S, Möhlenbruch MA, et al. Minor stroke syndromes in large-vessel occlusions: mechanical thrombectomy or thrombolysis only? AJNR Am J Neuroradiol 2017;38:1177-9.

49 Kastrup A, Brunner F, Hildebrandt $\mathrm{H}$, et al. Endovascular therapy versus thrombolysis in patients with mild strokes and large vessel occlusions within the anterior circulation. Interv Neurol 2018:7:431-8.

50 Heldner MR, Chaloulos-lakovidis P, Panos L, et al. Outcome of patients with large vesse occlusion in the anterior circulation and low NIHSS score. J Neurol 2020;267:1651-62.

51 Shang X, Lin M, Zhang S, et al. Clinical outcomes of endovascular treatment within 24 hours in patients with mild ischemic stroke and perfusion imaging selection. AJNR Am J Neuroradiol 2018:39:1083-7.

52 Kaesmacher J, Chaloulos-lakovidis P, Panos L, et al. Clinical effect of successful reperfusion in patients presenting with NIHSS. J Neuro/ 2019;266:598-608.

53 Dibajnia $\mathrm{P}$, Morshead CM. Role of neural precursor cells in promoting repair following stroke. Acta Pharmaco/ Sin 2013:34:78-90.
54 Parent JM, Vexler ZS, Gong C, et al. Rat forebrain neurogenesis and striatal neuron replacement after focal stroke. Ann Neurol 2002;52:802-13.

55 Alqahtani SA, Stemer AB, McCullough MF, et al. Endovascular management of stroke patients with large vessel occlusion and minor stroke symptoms. Cureus 2017;9:e1355.

56 Tejada Meza H, Martínez García R, Capablo Liesa JL, et al. Anticipating disaster: mechanical thrombectomy in patients with low NIHSS scores. Neurologia 2019;34:350-1.

57 Desai SM, Rocha M, Starr M, et al. Laterality is an independent predictor of endovascular thrombectomy in patients with low National Institute of Health Stroke Scale. J Stroke Cerebrovasc Dis 2018;27:3172-6.

58 Yang $X$, Wang D. Letter to the editor regarding "endovascular mechanical thrombectomy in large-vessel occlusion ischemic stroke presenting with low National Institutes of Health Stroke Scale: systematic review and meta-analysis". World Neurosurg 2019;125:542.

59 Yang P, Zhang Y, Zhang L, et al. Endovascular thrombectomy with or without intravenous alteplase in acute stroke. N Engl J Med 2020;382:1981-93.

60 Lee VH, Thakur G, Nimjee SM, et al. Early neurologic decline in acute ischemic stroke patients receiving thrombolysis with large vessel occlusion and mild deficits. J Neurointerv Surg 2020. doi:10.1136/neurintsurg-2020-015871. [Epub ahead of print: 10 Apr 2020].

61 Miteff F, Levi CR, Bateman GA, et al. The independent predictive utility of computed tomography angiographic collateral status in acute ischaemic stroke. Brain 2009;132:2231-8 